\title{
REVIEW
}

Open Access

\section{Behaviour change approaches for individuals with diabetes to improve foot self-management: a scoping review}

\author{
Joanne Paton ${ }^{1 *}\left(\mathbb{D}\right.$, Sally Abey ${ }^{1}$, Phil Hendy ${ }^{1}$, Jennifer Williams ${ }^{1,2}$, Richard Collings ${ }^{1,2}$ and Lynne Callaghan ${ }^{3}$
}

\begin{abstract}
Background: Diabetes related foot complications are increasing in complexity, frequency and cost. The application of self-management strategies can reduce the risk of individuals developing foot complications. The type, range and nature of the literature focusing on interventions that support patients with diabetic foot self-management is unknown. This scoping review aimed to i) identify self-management actions and risky behaviour avoidance strategies within interventions, ii) map the theoretical functions through which these behaviour change interventions have an effect, iii) display gaps in the research.

Methodology: Arksey and Malley's (2003) 5 stage framework was followed to conduct the scoping study. This methodological framework was selected because it was developed specifically for scoping reviews and therefore offered clear methodological distinction from systematic review methodology. .

Databases were searched from inception of the project until June 2020 supplemented by hand searching of reference lists. In total 988 papers were identified. These were independently screened by three reviewers, identifying 19 eligible papers. Data extraction and charting of data was independently conducted by three reviewers to identify study characteristics, self-management actions and risky behaviours. Data was charted against the COM-B (capability, opportunity, motivation, behaviour) model of behaviour to determine intervention function.

Results: In total 25 different foot self-management actions and risk behaviours were classified into three themes; routine self-management, trauma avoidance and warning signs and actions. Inspect feet daily received the most attention. The majority of interventions focused on knowledge and skills, but overlooked taking action and decision making. Intervention mapping identified four primary intervention functions (education, persuasion, training and enablement) used to address deficits in capability, opportunity and motivation that positively improved foot selfmanagement behaviour. No studies targeted first ulcer prevention, and most either did not measure or improve foot health outcomes.
\end{abstract}

Conclusion: This review charted the evidence for interventions promoting diabetic foot self-management through a theoretical behaviour change perspective. A core set of behaviour change activities and intervention functions associated with positive changes in behaviour were identified. This information will provide researchers with a useful basis for developing self-management interventions.

Keywords: Diabetic foot, Self-management, Behaviour change wheel, Foot ulcer prevention

\footnotetext{
* Correspondence: joanne.paton@plymouth.ac.uk

${ }^{1}$ School of Health Professions, Faculty of Health, University of Plymouth,

Derriford Road, Plymouth PL6 8BH, UK

Full list of author information is available at the end of the article
}

(c) The Author(s). 2021 Open Access This article is licensed under a Creative Commons Attribution 4.0 International License, which permits use, sharing, adaptation, distribution and reproduction in any medium or format, as long as you give appropriate credit to the original author(s) and the source, provide a link to the Creative Commons licence, and indicate if changes were made. The images or other third party material in this article are included in the article's Creative Commons licence, unless indicated otherwise in a credit line to the material. If material is not included in the article's Creative Commons licence and your intended use is not permitted by statutory regulation or exceeds the permitted use, you will need to obtain permission directly from the copyright holder. To view a copy of this licence, visit http://creativecommons.org/licenses/by/4.0/. The Creative Commons Public Domain Dedication waiver (http://creativecommons.org/publicdomain/zero/1.0/) applies to the data made available in this article, unless otherwise stated in a credit line to the data. 


\section{Key message}

- We recommend a more targeted, systematic approach to increase our understanding of the effectiveness of individual or clustered related foot care activities.

- Our exploratory findings may inform future intervention development. Clinically meaningful improvements in diabetic foot health seem to be associated with two combinations of intervention functions: 1. diabetic foot care education and foot care skills training delivered in combination with enablement (providing foot care equipment, prompts like daily diaries) Or 2. Diabetic foot care education and foot care skills training delivered in combination with persuasion (information to illicit an emotional response around what is good or bad foot care behaviour).

- Further research underpinned by a theoretical behaviour change framework, will help determine which combination of intervention functions are effective and cost-effective in supporting foot selfmanagement behaviour for preventing foot ulceration.

\section{Introduction}

Diabetes is a common long-term condition within the United Kingdom (UK) [1]. It is estimated that by 2025 the prevalence of diabetes will have doubled, from 2.7 million (2017) to over five million people [2, 3]. Every year, the National Health Service (NHS) undertakes over 7000 diabetic related lower limb amputations [4]. Eighty percent of these are preceded by preventable foot ulceration [5]. Fifty percent of people with diabetes who suffer a foot ulceration will not live beyond 5 years [6]. Between 31 and $36 \%$ of the diabetic population are classified at moderate or high-risk of developing a foot ulcer $[3,7]$. Medical resources are concentrated on those with pre-existing foot pathology. Long-term foot health outcomes for people with diabetes could be improved through diabetic self-management strategies targeted toward people with diabetes, at risk of foot ulceration.

Diabetes UK, and the James Lind Alliance Priority Setting Partnership, identified 'self-management in people with type 2 diabetes' as a research priority [8]. The umbrella term 'diabetic foot self-management' includes a range of behaviour related self-management actions and risk-taking behaviour modifications that individuals are advised to perform on a regular basis. Most of the research evidence is focused upon those with diabetes required to self-manage their blood sugar control [9]. As the burden of diabetic complications, including foot ulceration, begins to outweigh the threat of poor glycaemic control, the focus has begun to shift toward strategies of self-management for diabetic foot ulcer prevention [10].

Engaging people with type 2 diabetes to take an active role in their daily foot self-management is important to promote good diabetic foot health, protect against diabetic foot ulceration and obtain early treatment for new foot ulceration [5]. Foot self-management involves foot health maintenance such as cutting nails regularly or drying between the toes [11-16]. Risk prevention measures include activities such as never walking barefoot, wearing shoes that fit, and foot health monitoring tasks, for example, self-screening for foot problems and contacting an appropriate health professional in the event of an injury [11-14, 17-20]. Some elements of foot selfmanagement may be important for ulcer prevention and the maintenance of foot health. Diabetic foot selfmanagement programmes are available to educate, support and enable people at moderate or high risk of developing a foot ulcer to alter their behaviour and selfmanage their feet, however, uptake is low [21, 22].

Unfortunately many people with type 2 diabetes find it difficult to alter their behaviour and adhere to selfmanagement regimes, often with devastating consequences to foot health. Reasons for none adherence are complex, such as patients considering foot care a lower priority than medication adherence or blood glucose control [23]. There is a misconception amongst those at greatest risk, that an absence of symptoms signals that nothing can be wrong [24]. Individuals with diabetic neuropathy often present with a dangerous lack of protective pain perception [25]. It is often difficult for individuals to comprehend their daily exposure to risk of tissue damage, or the potential benefit of foot care selfmanagement [26] For many, it is only after experiencing the devastating consequences of diabetic foot ulceration first hand, that they become worried enough about their future foot health to engage in self-management to protect their feet for the future [23]. However, at this stage ulcer reoccurrence is often unavoidable. Approximately $40 \%$ of patients re-ulcerate within 1 year of their first ulcer, whilst $60 \%$ suffer a second ulceration within 3 years [27].

This scoping review maps the range of foot selfmanagement behaviours included within diabetic foot self-management interventions investigated within the effectiveness studies.

The second part of the review employs the COM-B framework [28] for behaviour change to characterise and compare the content of the diabetic foot selfmanagement interventions (Fig. 1). The widely used COM-B framework was developed through the systematic review and selection of relevant aspects of other behaviour change tools. The COM-B framework was considered the most suitable framework for this review 


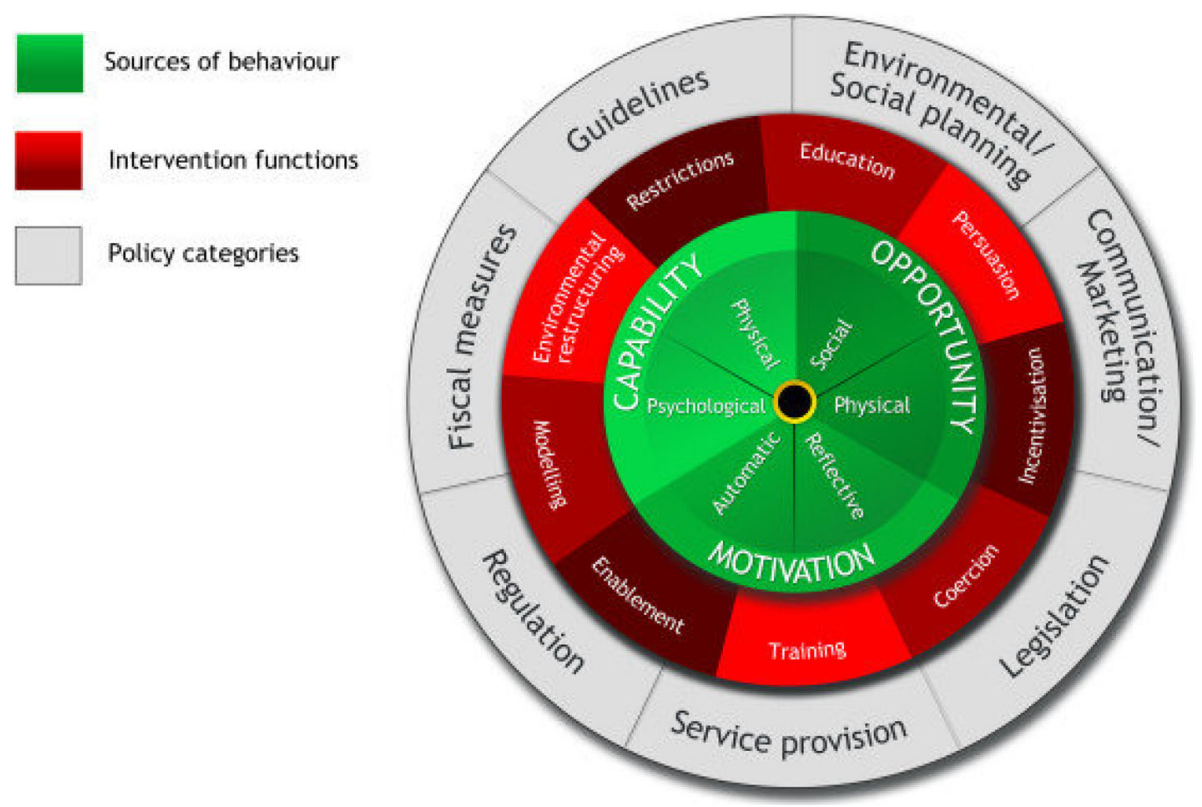

Fig. 1 Behaviour Change Wheel COM-B Framework of Behaviour Change [29]

because of its dual purpose; 1 . Providing explanation for why a recommended behaviour or intervention has not been implemented 2. Identifying domains that might be targeted as levers for change to inform intervention design. The psychology based COM-B model hypothesises that interaction between three components 'capability', 'opportunity' and 'motivation', best explains the variation in the success of the interventions devised to provoke change behaviour [10]. Through mapping the components of an intervention this review seeks to identify explanations for why a recommended behaviour is not implemented by an individual and identify domains that might inform the development of more effective interventions to support diabetic foot self-management by health professionals $[10,13]$.

\section{Aim}

The objective of this scoping review is first to identify which of the recommended foot self-management actions or risky foot health behaviours have been the focus of evaluation studies designed to test interventions to prevent ulceration in people with diabetes, and second to map the COM-B model and intervention functions used within those interventions.

Research Questions:

1. What are the self-management actions or risky behaviour modifications targeted by researchersevaluating behaviour change interventions for improving foot self-management for diabetic foot ulcer prevention and where are the gaps in research activity?

2. What are the specific intervention functions being implemented as part of the intervention and what conclusions have been drawn?

3. How are those interventions for improving foot self-management and reducing ulcer risk being implemented (context, target population, delivery format) and outcomes measured.

\section{Methods}

\section{Protocol and registration}

The protocol was drafted using the preferred reporting items for scoping reviews and meta- analysis protocols [30], and reviewed by the members of the research team. The protocol was registered retrospectively with the Open Science Framework on the 26th November 2019, (Registration number: osf.io/3ahsv).

The method employed to conduct the review followed the five stage framework described by Arksey and Malley [31] for conducting a scoping study and was reported in accordance with the PRISMA Extension for scoping reviews [32]. The inclusion and exclusion criteria for the scoping review was defined at the outset.

\section{Eligibility criteria Inclusion criteria}

The search was limited to articles written in English and adults over 18 years old, diagnosed with diabetes, without foot ulcer. Included were empirical studies evaluating the effectiveness of interventions directed towards 
the prevention of diabetic foot ulceration through the improvement of foot self-management or by the modification of risky foot health behaviour. Interventions reporting on outcome measures or analysis directed towards the modification of foot self-management behaviour were also included.

\section{Exclusion criteria}

Papers were excluded from the review if the intervention was directed at the healthcare providers behaviour, or intended for group delivery. Interventions intended for group delivery were considered outside of the scope of this review. The mode of delivery considered for inclusion within this review was selected to best reflect and therefore have increased relevance to the tailored approach to diabetic foot care commonly implemented clinical practice. Book reviews and commentaries were not included.

\section{Information sources}

Searches were conducted both electronically and manually. The search strategy comprised of three steps. First, an initial search was undertaken using the following electronic databases: MEDLINE, CINAHL, AMED, Embase, Cochrane Database of Systematic Reviews, PsycINFO. A second search for unpublished studies included Google Scholar. Finally, a manual search of reference lists was conducted. The reference lists of the selected articles were scanned to identify further studies of potential interest.

Search terms included Diabet* AND foot OR peripheral AND neuropathy, Self-management OR selfmanagement OR self-assessment OR patient centred (centered) OR education OR training OR behaviour* OR motivate*, Prevention AND ulcer* OR Foot AND protection OR adherence OR lifestyle change. The MEDL INE search strategy is provided as Additional File 1.

Three independent reviewers (JP, SA, PH) scanned the title and abstract of the articles identified by the searches to select those matching the inclusion criteria. Full texts of the remaining articles was retrieved. Any disagreement in the final selection of articles of data extraction was resolved through discussion.

\section{Data items}

The key information extracted from each paper was charted using a standardised data extraction tool developed and piloted for this study. The tool was used to capture information to describe the extent, nature and distribution of studies (for example, foot health actions targeted by the intervention, study context, research methods and outcomes, and recipient group characteristics). In addition, data relating to intervention characteristics (eg intervention type, intervention function, mechanism of action and evidence of effectiveness) was independently extracted and charted by the three reviewers. Disagreements were resolved through discussion between the three reviewers (JP, SA, PH) and by further adjudication by a forth reviewer (LC) with specific expertise in the pschcology of behaviour change.

\section{Collating, summarizing and reporting the results}

Studies were grouped to provide a broad view of the extent, nature and distribution of studies included within the review. This information was used to contextualise the data, and identify dominant areas of research activity and subsequently gaps in research activity.

Studies and intervention components specifying a behaviour change related to foot self-management were mapped thematically to classify the behaviour change. In addition, each intervention component was mapped and coded against the COM-B model of behaviour [29]. As anticipated at the outset, many interventions comprised of several sources of behaviour, and each source of behaviour served several intervention functions. In this situation, all sources of behaviour within an intervention were charted, but only the primary intervention function for each source of behaviour was captured. Once charted the data could be synthesised to determine the sources of behaviour and primary intervention functions shared between interventions found to be effective in changing behaviour to improve the foot self-management of people with diabetes.

\section{Results}

\section{Description of studies}

The PRISMA flow diagram details identification and selection of studies (Fig. 2). All citations, abstracts, and papers were independently scanned by three investigators (JP and SA or PH). The initial search produced 988 papers including 219 duplications. Screening by title and abstract of the remaining 769 citations identified 71 potentially relevant articles. Thirty-one articles were retrieved and 41 required further discussion. Of these, 21 papers were excluded and 20 full text articles retrieved. In total 51 potentially relevant full text articles were retrieved. Twenty-seven articles were excluded after reading full text. The primary reasons retrieved studies were excluded from the review were because the studies investigated group interventions. A further five papers were excluded at the data collection stage. A total of 19 papers reporting on 18 studies were included in the review.

\section{Mapping of results \\ Geographical distribution of studies}

The geographical distribution of studies shows the number and percentage of research reports that evaluated 


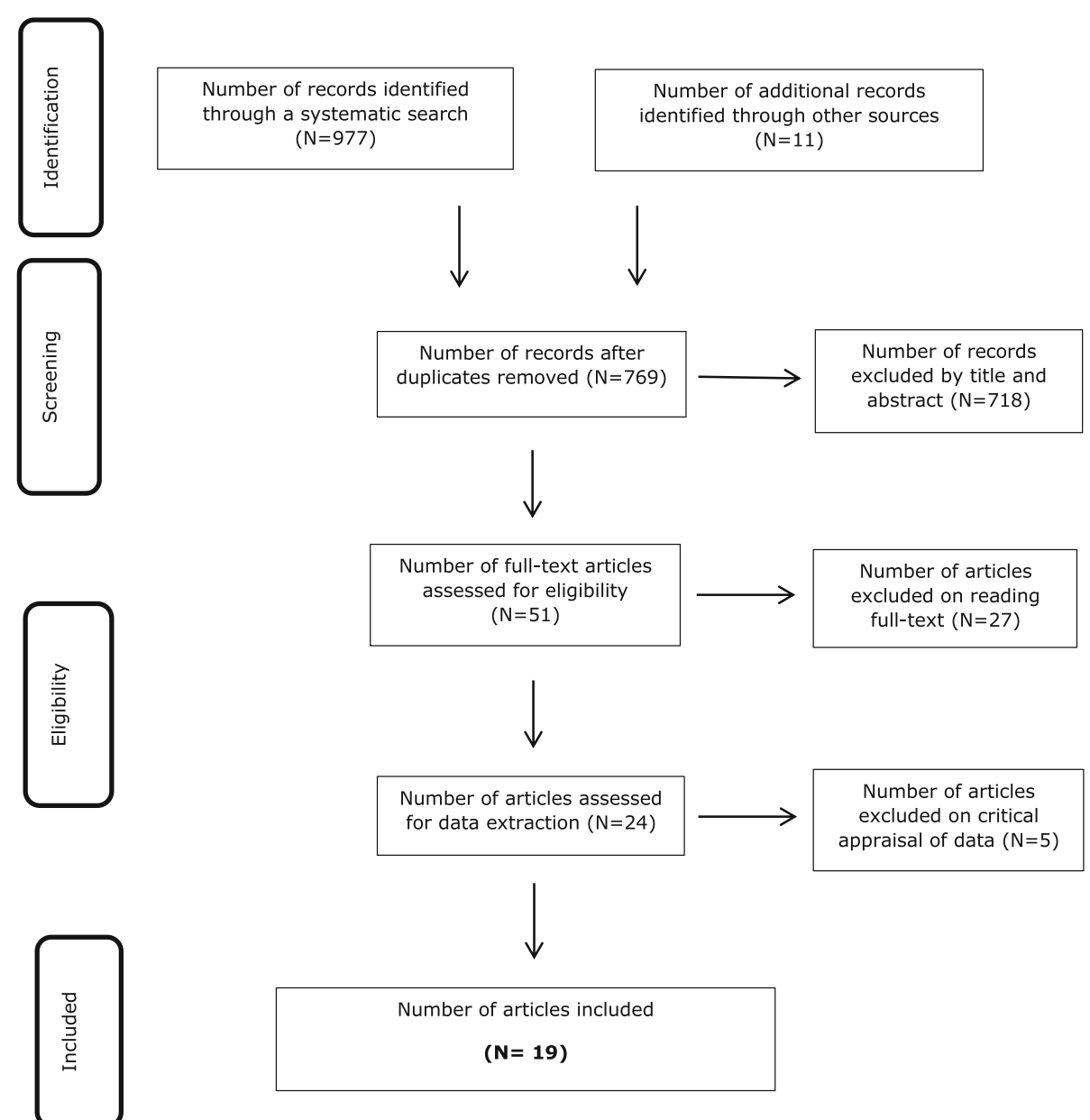

Fig. 2 PRISMA [33] flow diagram of search and study selection process

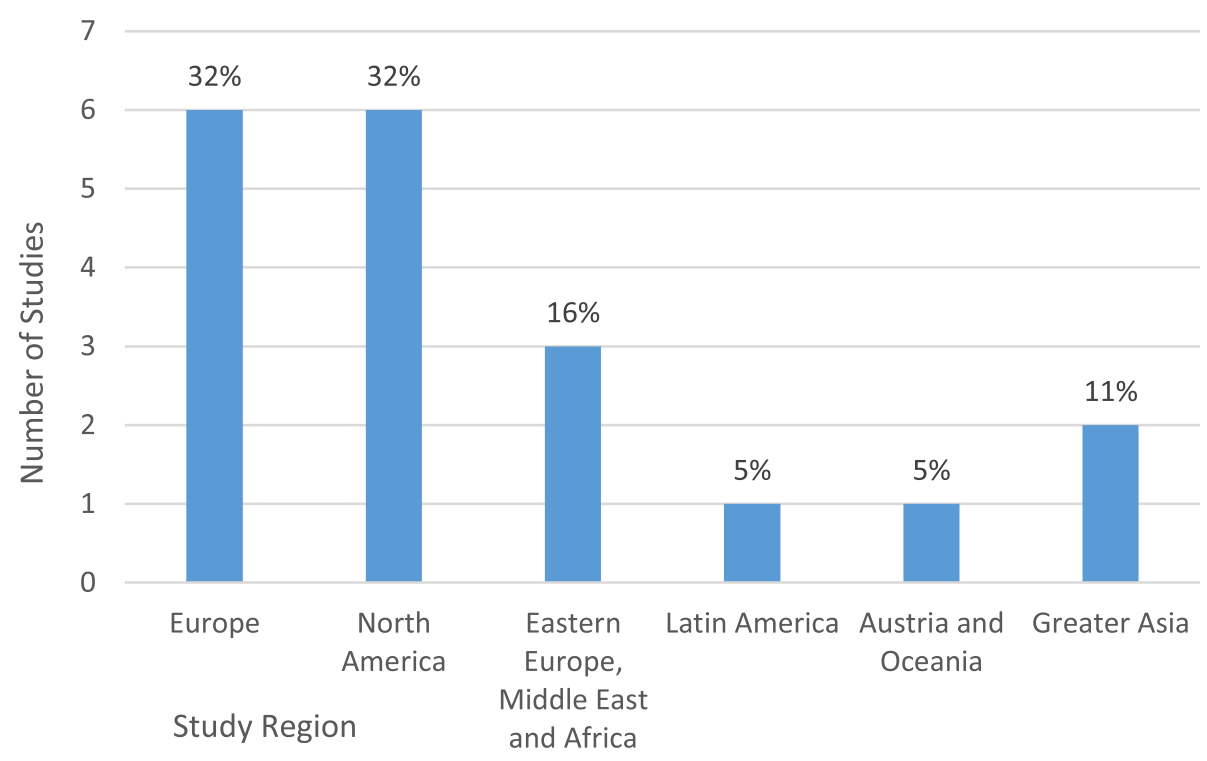

Fig. 3 Geographical distribution of studies 
interventions, to improve foot self-management behaviour for foot ulcer prevention in people with diabetes, according to the world region in which the intervention were implemented (Fig. 3). The majority of papers described interventions were carried out in North America (32\%) $[11,14,15,19,34]$ or Europe [12, 17, 18, 20, 35, 36] (32\%), including a single study from the UK [36]. Iran and Turkey accounted for the $16 \%$ of studies in the Eastern Europe Middle East and Africa Region [16, 37]. A Latin America study was implemented in Brazil [13]. China accounted for the two studies implemented in Asia [38, 39].

\section{Care setting distribution of studies according to recipient risk of ulceration}

Figure 4. shows the number and percentage of studies categorised by study participant diabetic foot ulcer risk status. Three studies (18\%) did not report the diabetic foot ulcer risk of the recipients recruited [12, 16, 34]. Emphasis on improving the foot self-management behaviour for foot ulcer prevention in those at high risk of diabetic foot ulceration was evident in $42 \%$ of the studies $[17,19,20,35,36,38-40]$. All studies recruiting highrisk participants were conducted within the Hospital setting $[17,19,20,38-40]$ (Table 1). However, the majority of studies $(80 \%)$, where recipients from the high risk category were excluded, were based within the community $[11,14,15,18]$. No studies were identified with specific focus on participants at risk of a first foot ulceration. The mean age of study participants categorised as low and increased risk for foot ulceration was 55 years. Study recipients at high risk of ulceration tended to be older (mean 63 years).
Type of research methods used to evaluate interventions to improve foot self-management in people with diabetes

Most studies (93\%) used a quantitative methodology. Of these, twelve of the studies used a randomised control trial design [12, 14, 17, 19, 20, 34, 36-38, 40-42], whilst five were uncontrolled before and after studies [11, 13, $16,35,39]$. Just one study used mixed methods employing quantitative outcome measures alongside semistructured interview [15].

\section{Sample size}

The sample size of the intervention group recruited to the studies varied considerably irrespective of research design with one RCT recruiting 5 participants [17] and another recruiting 267 [18]. The mean number of participants enrolled into the intervention group was 86 .

\section{Measures of effectiveness}

The outcome measures focussed on levels of knowledge, change in behaviour and foot health. The majority of studies developed their own scales to measure levels of knowledge [12, 14, 15, 18, 34, 38, 39], technical competence to carry out a skill or log the regularity with which a desired foot care behaviour was conducted [17, 19, 20, $35,36,40,42]$. A small number of studies used standardised 'off the shelf' measures of self-efficacy [14, 34, 39]. Half of all studies $(n=9)$ included a measure of foot health [13, 19, 20, 35-37, 40, 42, 43]. Measures of foot health most often concentrated on improved foot function or incidence of foot ulceration $[13,19,20,35-38$, $40,42]$. One study recorded the number of minor skin lesions as measure of effectiveness [43].

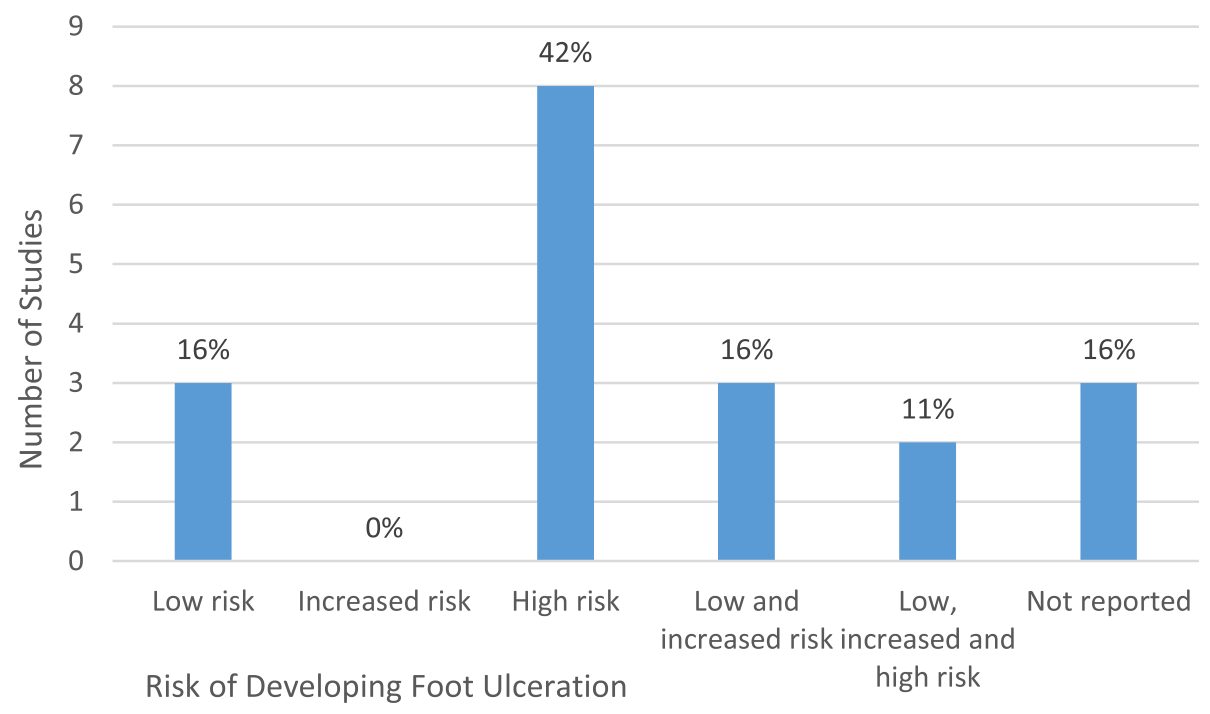

Fig. 4 Number and proportion of studies categorised according to participant risk of foot ulceration 
Table 1 Care setting distribution of studies according to recipient risk of ulceration

\begin{tabular}{lllll}
\hline & Low & Low and Increased & High & Not reported \\
\hline Hospital & & & 6 & 2 \\
Community & 2 & 4 & 1 & 2 \\
Home & & & 1 & \\
University Clinic & & 1 & & \\
\hline
\end{tabular}

\section{Categorising the intervention}

The category definitions were taken from the literature [44], to differentiate between interventions that deliver traditional patient education and those that provide selfmanagement education. Traditional patient education was defined as the provision of disease-specific information or skills intended to increase patient compliance through improved technical knowledge and skills (for example, how to cut toe nails safely or avoid walking without shoes). Self-management education in contrast, was defined as the provision of skills necessary to act on problems to improve health outcomes through increased self-efficacy (for example, how to self-monitor feet, understand when a condition is medically serious, how to seek help, and have the confidence to take action). Most foot self-management interventions (56\%) delivered traditional foot health education information and skills [12, 13, 17, 34, 35, 37, 38, 41, 42] (Table 2). One study provided self-management education only [19]. In this study, participants were taught how to self-monitor and log skin temperature and when they should reduce activity levels and contact a professional. The remaining studies (38\%) used a blend of education strategies to include diabetic foot care education, alongside managing skills (such as, inspecting feet daily and when to seek professional help) [14-16, 20, 38, 39, 43, 45].

Interventions were categorised as theory driven such as, intervention development underpinned by a conceptual behaviour change model or delivered applying the principles of health coaching. In this context the definition for health coaching was taken from the Better Conversation, Health Coaching website supported by the NHS Innovation Accelerator Programme (https://www. betterconversation.co.uk/). A health coaching approach is defined as a patient-centred process. It entails goal setting determined by the patient, encourages selfdiscovery, in addition to content education, and incorporates mechanisms for developing accountability in health behaviours. Only three studies (19\%) explicitly embraced this patient-centred approach to improving diabetic self-management (Table 2) [17, 34, 43]. One used motivational interviewing to facilitate increased internal motivation within participants, to increase insole adherence [17]. The other two were grounded within the conceptual framework of the Social Cognitive Theory $[34,43]$. Both interventions were designed to change foot care behaviour through improved diabetes selfefficacy.

\section{Categorising foot health actions (Table 3)}

There were many different foot health actions incorporated within the interventions included in this review. Despite the review being based on just 18 research studies, a total of 25 different foot health actions were evaluated. Once a list of foot health actions had been extracted from the literature, to organise the data more meaningfully and to better identify trends or gaps in the evidence, foot health actions were grouped into three broad behaviour orientated headings through a process of theming:: 1 . routine self-management, 2 . trauma avoidance and foot protection, 3. warning signs and taking action. Those interventions targeted at improving routine self-management (for instance 'wash and dry feet daily') were more likely to be delivered as measurable goals or skill-based tasks. In contrast interventions focused on reducing risky behaviour for trauma avoidance or foot protection tended to be framed in more general terms (for instance 'wear shoes that fit'). Eleven studies (over 60\%) were aimed at improving self-screening for early signs of foot problems $[11,13,14,16,18,19,34$, $36,38,39$ ] (Table 3). Several of these failed to recognise that improved self-screening is likely to have limited beneficial impact on health outcomes unless combined with associated self-management foot health activities such as decision making and taking action. For example whilst four studies included self-screening, authors omitted to include the critical decision making element around 'when and how to seek help' [13, 34, 38, 39]. Likewise, on finding early signs of foot problems, all but two failed to include any sort of damage limitation activity such as 'reducing activity levels' $[19,20]$.

Most studies (68\%) included interventions addressing foot health actions from two or more of the categories

Table 2 Number and proportion of studies according to intervention category

\begin{tabular}{|c|c|c|c|c|}
\hline \multirow[t]{2}{*}{ Categories } & \multicolumn{2}{|c|}{ All studies } & \multicolumn{2}{|c|}{ Theory driven interventions } \\
\hline & N (18) & $\%$ & N (3) & $\%$ \\
\hline Traditional patient education & 10 & 56 & 1 & 6 \\
\hline Self-management education & 1 & 6 & 0 & - \\
\hline Both & 7 & 38 & 2 & 13 \\
\hline
\end{tabular}




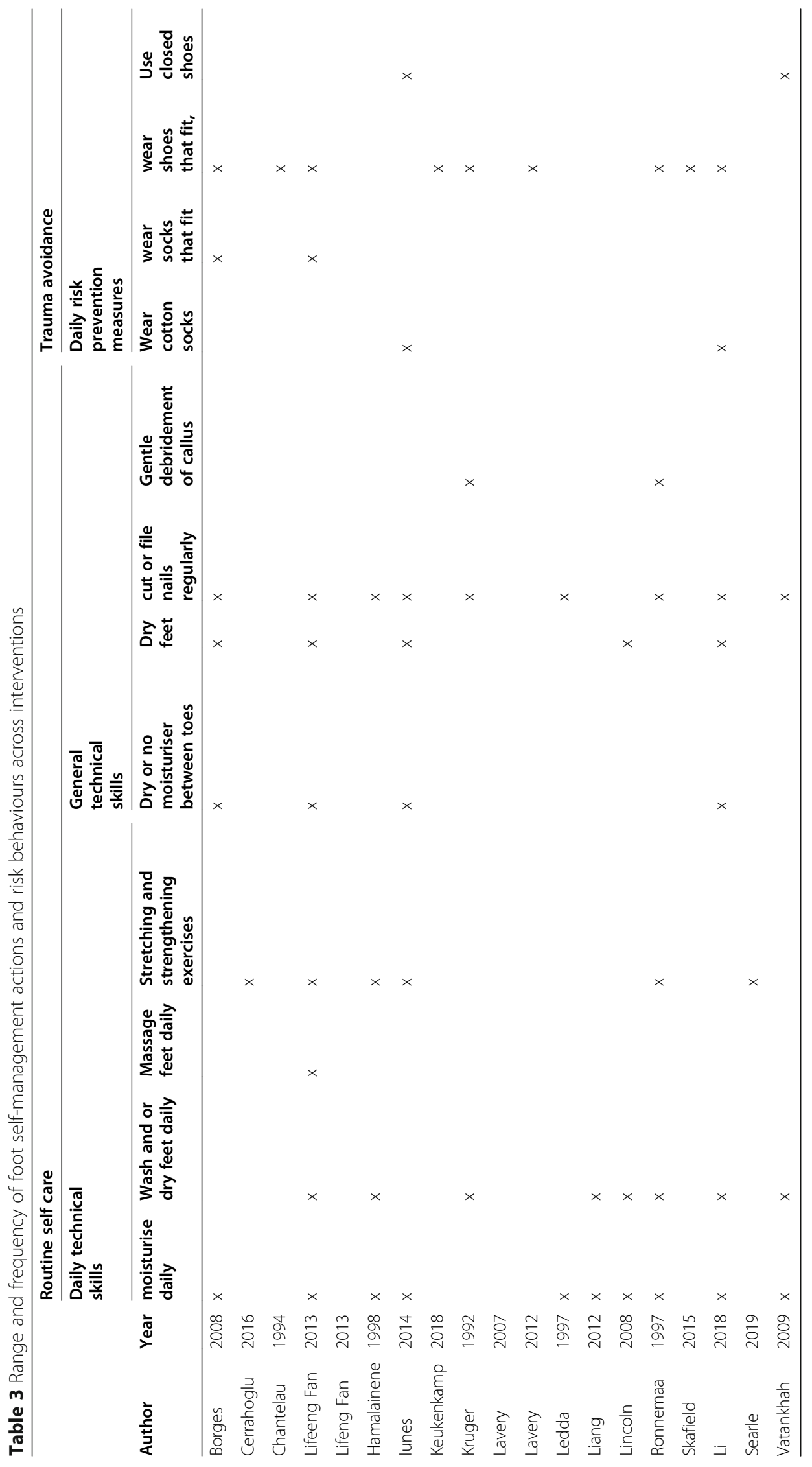




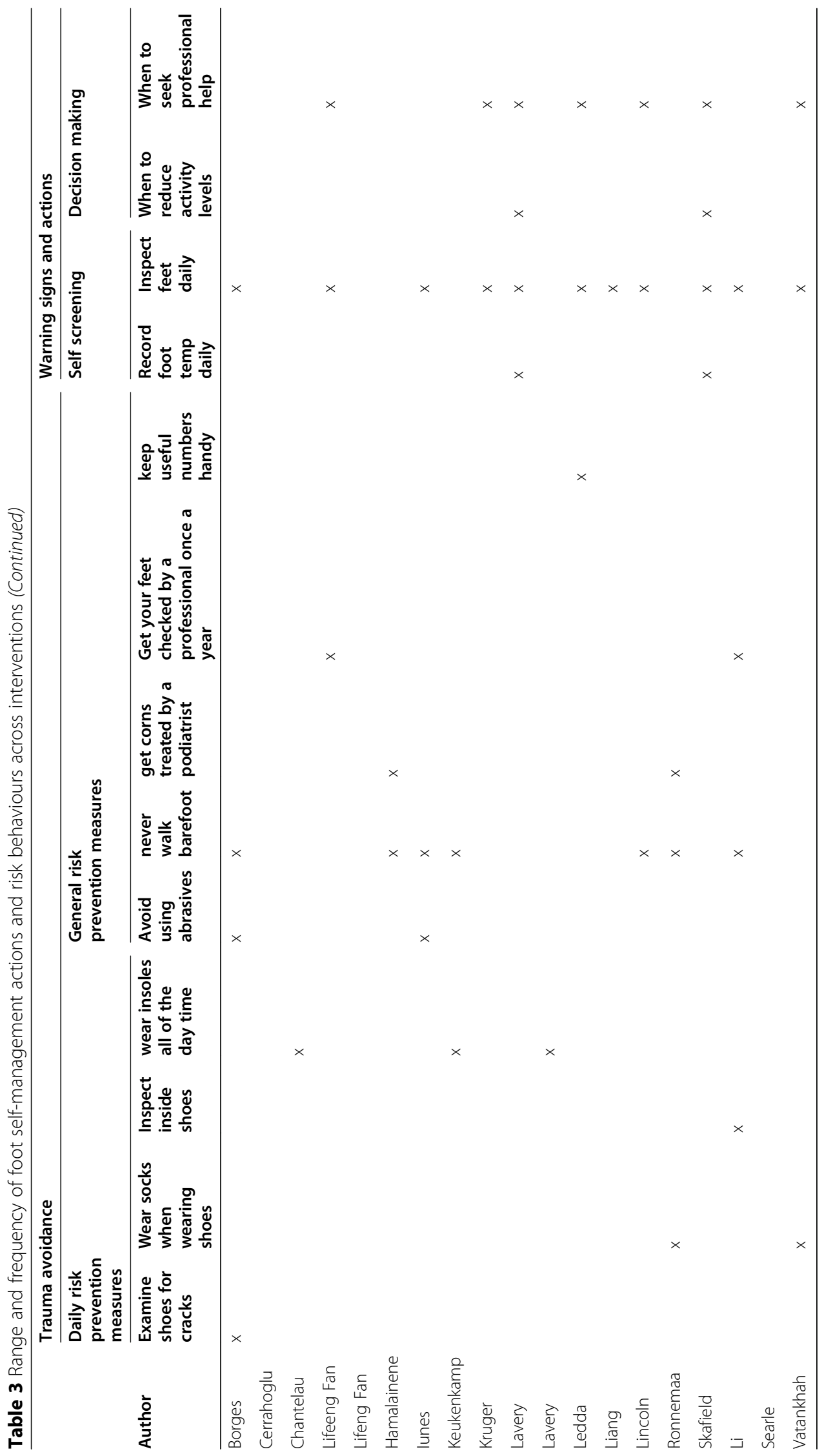


$[11-16,18,20,34,36,38,39]$. The intervention implemented in eight of these studies comprised of a comprehensive foot self-management improvement package, encompassing foot health actions from all three categories (routine self-management, trauma avoidance and foot protection, warning signs and decision making) [11, 13$16,34,36,39]$. The number of foot care actions combined within each of these interventions ranged from five to twelve. Twenty-five percent of studies took a more focused approach to behaviour change intervention design, targeting just one category of foot health activities $[17,19,35,37,40,42]$. The number of activities combined within each of these interventions ranged from one to four. The type of foot health activities associated with this more focused intervention approach were largely aimed at increasing participant adherence to medical devices, specifically therapeutic footwear and insoles for foot protection and skin thermometers for self-monitoring changes in foot temperature $[17,19,35,40]$.

The most frequently targeted and therefore arguably the most important foot health action across all categories was 'inspect feet daily'. The remaining most frequently targeted foot health actions were all positioned with the routine care category and included moisturise feet daily, wash and dry feet daily, cut or file nails regularly. 'Wear shoes that fit' and the inversely phased 'never walk barefoot' appeared to be the only foot health activity with consensus between studies from the trauma avoidance and foot protection category. Within this category there were a number of foot health activities with a low rate of occurrence. Most of these were inconsistent foot health activities related to sock choice or fit.

\section{Mapping intervention functions and sources of behaviour change}

The COM-B model provides a systematic method of identifying nine possible intervention functions enabling a given intervention or target behaviour (Fig. 1) [28]. Intervention functions were identified and compared across studies to determine which were most commonly employed in footcare self-management interventions [28]. Figure 5. Show the frequency of intervention functions across all interventions for foot protection. Four intervention functions were clearly better suited to this type of behaviour change intervention. Education: increasing knowledge or understanding. Persuasion: Using communication to induce positive or negative feelings or stimulate action. Training: Imparting skills. Enablement: Increase means to increase capability.

The intervention functions form part of the COM-B as a framework for understanding behaviour. Each intervention function can be mapped against one of three essential sources of behaviour (capability, opportunity and motivation). Interventions change behaviour by addressing deficits in one of more of these interacting sources of behaviour (Fig. 1) [28].

Figure 6 shows that capability (Psychological and Physical) were behavioural deficits most frequently addressed by interventions.

The table shows the frequency of intervention functions mapped against the COM-B model sources of behaviour across all interventions for foot protection (Table 4). Capacity-Psychological/Education and Capacity-Physical/ Training were the intervention function and source of behaviour used with most frequency within interventions for foot protection. This was predominantly in combination

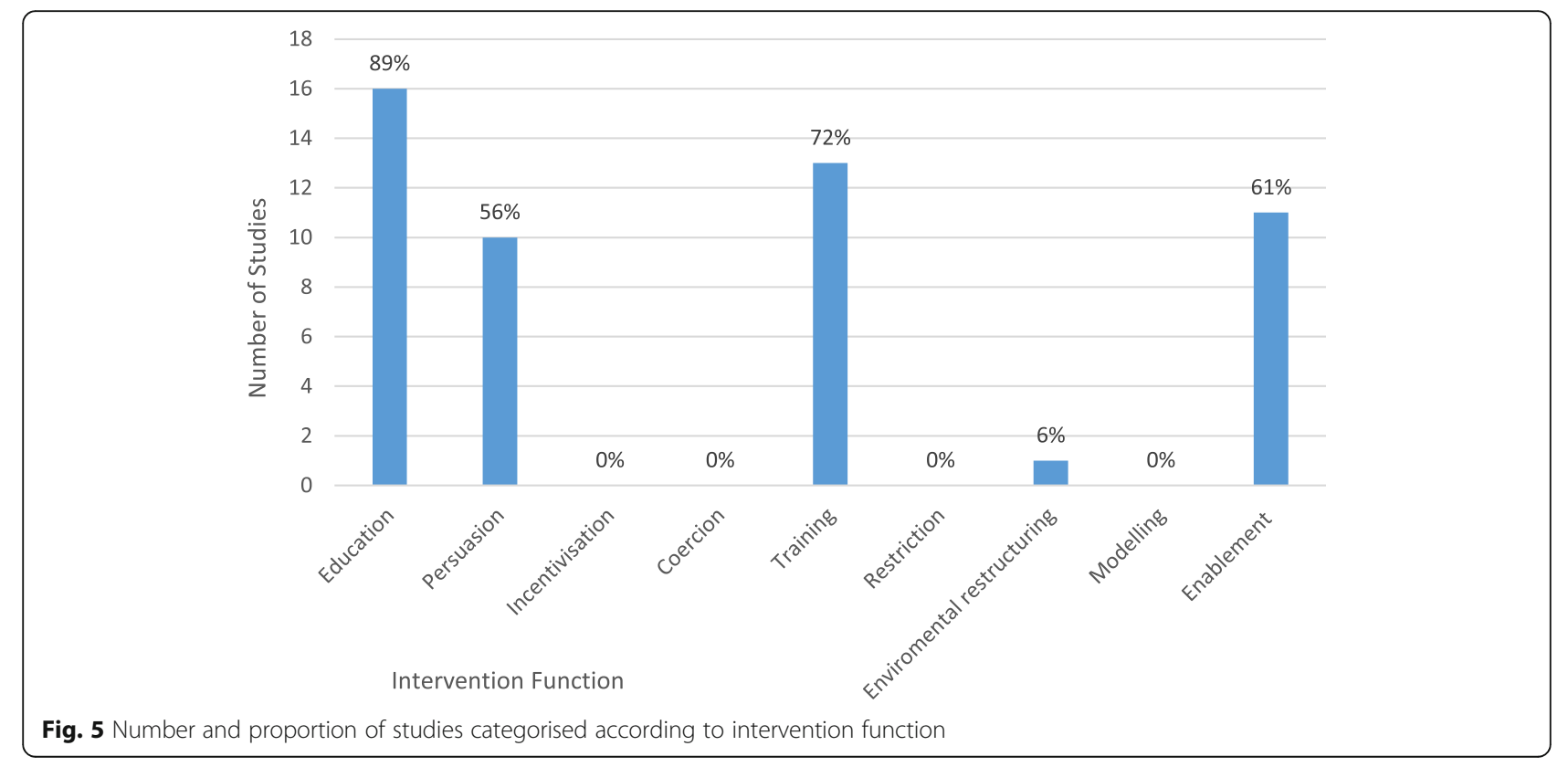




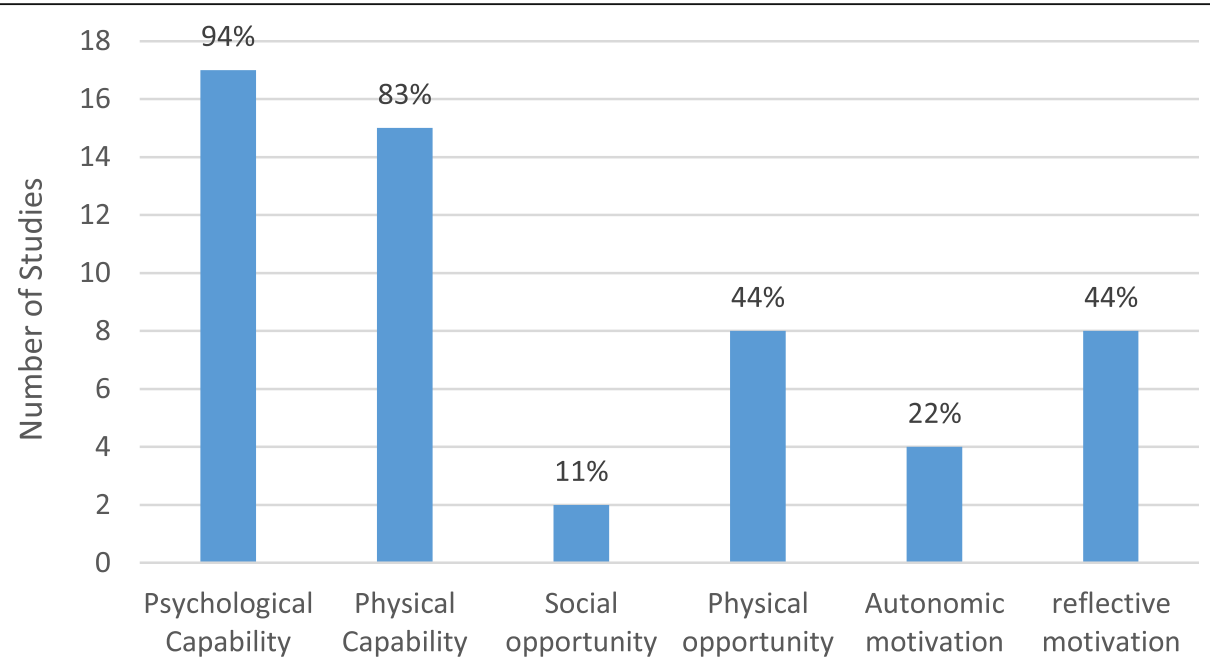

Source of Behaviour

Fig. 6 Number and proportion of studies categorised according to sources of behaviour. Detailed legend: The following descriptors are taken from Michie [29]. 1. Physical capability can be achieved through physical skill development with is the focus of training or potentially through enabling interventions such as medication surgery or prostheses. 2. Psychological capability can be achieved through imparting knowledge or understanding training emotional cognitive and / or behavioural sills or through enabling interventions such as medication. 3. Reflective motivation can be achieved through increasing knowledge and understanding eliciting positive (or negative) feelings about behavioural target. 4 . Autonomic motivation can be achieved through associative learning that elicit positive (or negative feelings and impulses and counter-impulses relating to the behavioural target, imitative learning, habit formation or direct influences on automatic motivational processes. 5. Physical and social opportunity can be achieved through environmental change [29]

with enablement to create Opportunity-Physical and/or persuasion to illicit Motivation-reflective. Sources of behaviour infrequently used were Opportunity (Social) and Motivation (Autonomic). Intervention functions never or rarely employed in interventions for foot protection included; incentivisation, coercion, modelling, restriction and environmental restructuring.

Sub-group behaviour change analysis was conducted on the very small number of interventions reporting a significant result, to determine which intervention functions are most effective in changing foot care behaviour. Interventions that improved participant knowledge all included education and enablement as the intervention function [12, 15, 16, 18, 34, 38]. Interventions that were successful in changing behaviour included education and either enablement training or persuasion $[12,16,17,19$, $20,34,36,38,39]$. Interventions that resulted in improvements in foot health all contained training and education with the addition of either persuasion or enablement $[18,19]$.

\section{Discussion}

The aim of this scoping review was first to identify the range of recommended foot self-management actions or risky foot health behaviour modifications for foot ulcer prevention, tested in evaluation studies. This information provides insight into areas of foot self-management with an evidence-base to support its efficacy and identify those areas where to date no research has been conducted. The second part of this review was designed to map the component parts of each foot self-management intervention against the COM-B framework, to define the function of the intervention in terms of its source of behaviour change. In addition, the mapping exercise

Table 4 Frequency of intervention functions mapped against sources of behaviour across all interventions for foot protection

\begin{tabular}{|c|c|c|c|c|c|c|c|c|c|}
\hline $\begin{array}{l}\text { Model of } \\
\text { behaviour; sources }\end{array}$ & Education & Persuasion & Incentivisation & Coercion & Training & Restriction & $\begin{array}{l}\text { Environmental } \\
\text { restructuring }\end{array}$ & Modelling & Enablement \\
\hline C-Ph & & & & & 21 & & & & \\
\hline C-Ps & 19 & & & & 4 & & & & 2 \\
\hline M-Re & 2 & 13 & & 0 & & & & & \\
\hline $\mathrm{M}-\mathrm{Au}$ & & 0 & 0 & 0 & & & 1 & 0 & 3 \\
\hline O-Ph & & & & & 1 & 0 & 1 & & 10 \\
\hline O-So & & & & & & 0 & 0 & & 2 \\
\hline
\end{tabular}


provides context information regarding geographical location, healthcare setting, healthcare provider of the included study, details of the recipient group, in terms of diabetes disease severity, and the nature of the studies, including research methods adopted and measures of effectiveness.

Most of the included studies took a 'scattergun' approach to improving foot care self-management by electing to evaluate multidimensional interventions, specifying up to 12 behaviours $[12-16,18,20,34,36,38]$ which makes determining the value of each variable to ulcer prevention impossible. Many interventions failed to target the correct behaviour that needed to change to prevent foot ulceration. Whilst ten studies provided education on selfscreening for warning signs for foot problems [11, 13, 14, $16,18,34,36,38]$, only two included the necessary decision-making, and action to be taken, as part of the target behaviour [13, 34]. Most emphasis was placed on ulcer detection rather than prevention. Twelve interventions included elements on reducing risky behaviour for trauma avoidance for foot protection (three targeted wearing insoles) $[11,13-16,18,34,36]$. However, the majority of behaviours were ill-defined. For example, cutting toe nails frequently and wearing shoes that fit. 'Inspect feet daily' received greatest attention from the literature.

Interventions were often aimed at those at high risk of foot ulceration $(41 \%)[19,20,35,36,38,40]$ or included people from the low risk category $(36 \%)[11,14,15,18]$. The remaining studies did not specify participant diabetic foot ulcer risk status within their report. No studies targeted people with diabetes at risk of developing a first foot ulceration. Outcome measures were not comparable between studies. However, seven studies (41\%) used participant knowledge levels as a measure of intervention effectiveness [11, 12, 14, 16, 18, 34, 38]. Incidence of ulceration, amputation, incidence of minor foot problems, and prevalence of foot conditions, foot temperature and visits to the podiatrist were outcome measures all used to assess foot health $[11,18,20,35,36,38,40]$. No studies included an economic component. To achieve clinical impact, future definitive randomised control trials should include incidence of ulceration as their primary outcome. Secondary short-term outcome measures such as knowledge levels and behaviour change are useful to give insight into the mechanism of action for intervention development, and as an indicator of intervention potential prior to proceeding an intervention to a full trial. Authors should be encouraged to develop innovative approaches for the assessment of self-management interventions which draw upon the patient experience and expertise to ensure that interventions best meet the needs of people living with diabetes. In particular outcome measures which capture affordability, practicality of service delivery within clinical context, acceptability to patients to optimise attendance and adherence, and equity for patients with disparities in health and from different social sectors.

The majority of foot care self-management interventions $(88 \%)$ are delivered using didactic, professionalcentred health education [11-16, 18, 34, 36]. This tends to cover skill-based tasks such as self-screening, routine foot care and general hygiene. Whilst this approach can be successful in improving knowledge and foot selfmanagement skills, participant decision-making abilities around when to seek professional help or reduce activity levels was not seen to improve. Possibly because behaviour change determinants (capability, opportunity and motivation) were not being addressed in the intervention design.

Most papers lacked detail in their description of intervention content, method of development and intervention delivery. Only three studies evaluated foot care selfmanagement interventions developed using a behavioural change framework [17, 34, 46]. Only three studies embraced a patient centred approach to improving diabetic foot self-management and its delivery for foot ulcer prevention [17, 34, 43]. Research favouring a patient centred approach or underpinned by a theoretical behaviour change framework used 'change in participant behaviour' as the preferred outcome measure. Intervention delivery was inconsistent; sessions ranged from one 15min contact, to 13 contacts over a one-year period.

The evidence was charted against the COM-B model to reveal a core set of components from the behaviour change model best suited for the development of interventions to promote foot self-management. This core set of components are Psychological Capability (necessary knowledge), Physical Opportunity (necessary time and resources) and Reflective Motivation (intent and beliefs about consequences). These core components were inked to four intervention functions. The two key combinations of the four intervention functions identified as most likely to bring about a change in foot selfmanagement behaviour are 1. Education (increasing knowledge of their diabetes and foot self-management) and skills training (imparting physical foot selfmanagement skills and techniques) in combination with persuasion (using communication to induce positive feelings about foot management or negative feelings about foot ulceration as a consequences of undesirable behaviour), and 2. Education and skills training in combination with enablement (increasing capability or opportunity by providing cues, prompts or equipment).

\section{Gaps and recommendations for future research}

This review noted a number of potential areas for future research to advance the development of diabetic foot selfmanagement management interventions. Intervention 
development could be accelerated through the employment of robust, systematic approaches to intervention design. This would place emphasis on defining the specific foot care self-management behaviour for change, the impact of that change on ulceration risk and the extent to which the behaviour can be measured. This development work will require innovative approaches to design, implementation and assessment interventions, which draw upon the patient's experience and expertise.

To make a decision on best practice, randomised controlled trials implemented over an extended follow up period are required to determine which combination of behaviour change techniques, underpinned by a theoretical behaviour change framework, are effective, and costeffective, in supporting foot self-management behaviour for preventing foot ulceration. Clinical trials should use sound methodologies to examine the best way to put into practice (context, delivery format) and measure (outcome measures) foot care self-management interventions. The development and application of standard outcome measures to determine the effectiveness of an intervention for foot ulcer prevention would enable better evaluation and comparison.

\section{Limitations}

Several limitations for this scoping review should be acknowledged. Publications not in the English language were excluded, potentially reducing the available data. Additionally, although the main search bases, reference lists, biographies and google scholar were recruited, some of the grey literature may have been missed. Therefore, this review may not have exhausted all sources of data. It is also recognised that the research team lacked expertise in behaviour change, to reduce the risk of bias, a behavioural psychologist provided expert oversight, and mentorship for this area of the work.

\section{Conclusion}

The self-management actions or risky behaviour modifications targeted by most studies evaluating behaviour change interventions for improving foot selfmanagement for diabetic foot ulcer prevention appear to focus on an ad-hoc combination of self-screening and skill based foot care tasks. To increase impact on foot health outcomes intervention designers should shift emphasis toward empowering and building patient confidence to enable sound decision making around taking preventative action or seeking professional help when warning signs or ulceration are discovered.

With reference to the COM-B model, to understand behaviour, to have the greatest chance of success interventions to support diabetic foot self-management should be underpinned by behaviour change theory and aim to address all determinants of behaviour; capability, opportunity and motivation. Charting the evidence revealed a core set of intervention functions common to interventions effective in improving foot selfmanagement behaviour (education and training and at least one other: persuasion or enablement).

A number of research gaps were identified through conducting this review. Randomised controlled trials with an extended follow up are particularly required, targeting those yet to develop first diabetic foot ulceration. Development of a core set of minimum outcome measures for clinical trials for diabetic foot ulcer prevention are required to reduce the social and economic impact of foot ulceration globally.

\section{Supplementary Information}

The online version contains supplementary material available at https://doi. org/10.1186/s13047-020-00440-w.

\section{Additional file 1.}

Additional file 2.

Additional file 3.

Acknowledgments

Not applicable.

\section{Authors' contributions}

JP conceived the idea. JP, SA, PH and LC designed the study. JP, SA and PH carried out the literature search and extracted the data. Consensus was reached by JP, SA, PH and LC. JP, SA, RC and JW drafted the manuscript. All authors have authors have seen and approved the final manuscript.

\section{Funding}

Dr. Joanne Paton is funded by a National Institute for Health Research (NIHR), (Clinical Trials Fellowship) for this research project.

Richard Collings is funded by a National Institute for Health Research (NIHR), (Doctorial Clinical Academic Fellowship) for this research project.

Jenifer Williams is funded by a National Institute for Health Research (NIHR), (Pre Doctorial Clinical Academic Fellowship) for this research project.

This publication presents independent research funded by the National Institute for Health Research (NIHR). The views expressed are those of the author(s) and not necessarily those of the NHS, the NIHR or the Department of Health and Social Care.

\section{Availability of data and materials}

The datasets used and/or analysed during the current study are available from the corresponding author on reasonable request.

\section{Ethics approval and consent to participate}

Not applicable.

\section{Consent for publication}

Not applicable.

\section{Competing interests}

The authors declare that they have no competing interests.

\section{Author details}

${ }^{1}$ School of Health Professions, Faculty of Health, University of Plymouth, Derriford Road, Plymouth PL6 8BH, UK. ${ }^{2}$ Torbay \& South Devon NHS Foundation Trust, Torbay Hospital, Lowes Bridge, Torquay TR2 7AA, UK. ${ }^{3}$ Penninsula Medical School, Faculty of Health, University of Plymouth, John Bull Building Plymouth Science Park, Plymouth PL6 8BT, UK. 
Received: 17 July 2020 Accepted: 23 November 2020 Published online: 06 January 2021

\section{References}

1. Hex N, Bartlett C, Wright D, Taylor M, Varley D. Estimating the current and future costs of type 1 and type 2 diabetes in the UK, including direct health costs and indirect societal and productivity costs. Diabet Med. 2012 29(7):855-62.

2. National Diabetes Audit 2018 [Available from: https://digital.nhs.uk/dataand-information/publications/statistical/national-diabetes-audit.

3. Khanolkar MP, Bain SC, Stephens JW. The diabetic foot. QJM. 2008;101(9):685-95.

4. Kerr M, Rayman G, Jeffcoate WJ. Cost of diabetic foot disease to the National Health Service in England. Diabet Med. 2014;31(12):1498-504.

5. Bus SA, Netten JJ, van Netten JJ. A shift in priority in diabetic foot care and research: 75\% of foot ulcers are preventable. Diabetes Metab Res Rev. 2016; 32:195-200

6. Kerr M. Foot care for people with diabetes; an economic case for change. BMJ. 2012; [Available from: https://improve.bmj.com/improve_post/footcare-for-people-with-diabetes-the-economic-case-for-change/.

7. Leese GP, Stang D, Mcknight JA. A national strategic approach to diabetic foot disease in Scotland: changing a culture. Bri J Diab Vasc Dis. 2011;11(2):69-73.

8. Finer SRP, Cowan K, Daly A, Robertson E, Farmer A. Top ten research prioities for type 2 diabetes: results form the Diabetes UK - James Lind Alliance priority setting partnership. Lancet. 2017;5:935.

9. Zoffmann V, Kirkevold M. Realizing empowerment in difficult diabetes care: a guided self-determination intervention. Qual Health Res. 2012;22(1):103-18.

10. Gordis A, Scuffham P, Shearer A, Oglesby A, Tobian J. The health care costs of diabetic peripheral neuropathy in the US. Diabetes Care. 2003;26(6):1790-5.

11. Lifeng F, Souraya S, Angela C-B, Kelly M. Feasibility, acceptability and effects of a foot self-care educational intervention on minor foot problems in adult patients with diabetes at low risk for foot ulceration: a pilot study. Can J Diabetes. 2013;37(3):195-201.

12. Hamalainen H, Ronnemaa T, Toikka T, Liukkonen I. Long-term effects of one year of intensified podiatric activities on foot-care knowledge and self-care habits in patients with diabetes. Diabetes Educ. 1998;24(6):734-40.

13. lunes DH, Rocha CBJ, Borges NCS, Marcon CO, Pereira VM, Carvalho LC. Selfcare associated with home exercises in patients with type 2 diabetes mellitus. PLoS One. 2014;9(12):e114151.

14. Kruger S, Guthrie D. Foot care: knowledge retention and self-care practices. Diabetes Educ. 1992;18(6):487-90.

15. Ledda MA, Walker EA, Basch CE. Development and formative evaluation of a foot self-care program for African Americans with diabetes. Diabetes Educ 1997:23(1):48-51.

16. Vatankhah N, Khamseh ME, Noudeh YJ, Aghili R, Baradaran HR, Haeri NS. The effectiveness of foot care education on people with type 2 diabetes in Tehran. Iran Primary Care Diabetes. 2009;3(2):73-7.

17. Keukenkamp R, Merkx MJ, Busch-Westbroek TE, Bus SA. An explorative study on the efficacy and feasibility of the use of motivational interviewing to improve footwear adherence in persons with diabetes at high risk for foot ulceration. J Am Podiatr Med Assoc. 2018;108(2):90-9.

18. Ronnemaa T, Hamalainen H, Toikka T, Liukkonen I. Evaluation of the impact of podiatrist care in the primary prevention of foot problems diabetic subjects. Diabetes Care. 1997;20(12):1833-7.

19. Lavery LA, Higgins KR, Lanctot DR, Constantinides GP, Zamorano RG, Athanasiou KA, et al. Preventing diabetic foot ulcer recurrence in high-risk patients: use of temperature monitoring as a self-assessment tool. Diabetes Care. 2007;30(1):14-20

20. Skafjeld A, Iversen MM, Holme I, Ribu L, Hvaal K, Kilhovd BK. A pilot study testing the feasibility of skin temperature monitoring to reduce recurrent foot ulcers in patients with diabetes -- a randomized controlled trial. BMC Endocr Disord. 2015;15(1):1-7.

21. Mogre $\mathrm{V}$, Johnson NA, Tzelepis F, Shaw JE, Paul C. A systematic review of adherence to diabetes self-care behaviours: evidence from low- and middle-income countries. J Adv Nurs. 2019;75(12):3374-89.

22. HSD HTS, Gohdes D. Foot care practices, services and perceptions of risk among medicare beneficiaries with diabetes at high and low risk for future foot complications. Foot Ankle Int. 2001;22(9):734-8.

23. Coffey L, Mahon C, Gallagher P. Perceptions and experiences of diabetic foot ulceration and foot care in people with diabetes: a qualitative metasynthesis. Int Wound J. 2019;16(1):183-210.
24. Boulton AJM. What you can't feel can hurt you. J Vasc Surg. 2010;52(3 Suppl):28S-30S

25. Caselli A, Pham H, Giurini J, Armstrong D, Veves A. The forefoot to rearfoot plantar pressure ratio is increased in severe diabetic neuropathy and can predict foot ulceration. Diabetic Care. 2002;25:1066-71.

26. Paton JS, Roberts A, Bruce GK, Marsden J. Patients' Experience of therapeutic footwear whilst living at risk of neuropathic diabetic foot ulceration: an interpretative phenomenological analysis (IPA). J Foot Ankle Res. 2014;7(1):16.

27. Boulton A, Vileikyte L, Ragnarson-Tennvall G, Apelqvist J. The global burden of diabetic foot disease. Lancet. 2005:366(12):1719-23.

28. Michie S, van Stralen MM, West R. The behaviour change wheel: a new method for characterising and designing behaviour change interventions. Implement Sci. 2011;6(42):1-11.

29. Michie S, Atkins L, West R. The behaviour change wheel: a guide to designing interventions: London silverback publishing; 2014.

30. Moher D, Shamseer L, Clarke M, Ghersi D, Liberati A, Petticrew M, et al. Preferred reporting items for systematic review and meta-analysis protocols (PRISMA-P) 2015 statement. Syst Rev. 2015:4(1):1

31. Arksey H, O'Malley L. Scoping studies: towards a methodological framework. Int J Soc Res Methodol. 2005;8(1):19-32.

32. Tricco AC, Lillie E, Zarin W, O'Brien KK, Colquhoun H, Levac D, et al. PRISMA extension for scoping reviews (PRISMA-SCR): checklist and explanation. Ann Intern Med. 2018;169(7):467-73.

33. Moher D, Liberati A, Tetzlaff J, Altman D. The PRISMA Group (2009). Preferred Reporting Items for Systematic Reviews and Meta-Analyses: The PRISMA Statement. BMJ (open access). 2009;339:b2535. https://doi.org/10.1136/bmj.b2535.

34. Borges WJ, Ostwald SK. Improving foot self-care behaviors with pies Sanos. West J Nurs Res. 2008;30(3):325-41.

35. Chantelau $E$, Haage P. An audit of cushioned diabetic footwear: relation to patient compliance. Diabet Med. 1994;11(1):114-6.

36. Lincoln NB, Radford KA, Game FL, Jeffcoate WJ. Education for secondary prevention of foot ulcers in people with diabetes: a randomised controlled trial. Diabetologia. 2008;51(11):1954

37. Cerrahoglu L, Kosan U, Sirin TC, Ulusoy A. Range of motion and plantar pressure evaluation for the effects of self-care foot exercises on diabetic patients with and without neuropathy. J Am Podiatr Med Assoc. 2016; 106(3):189-200

38. Liang R, Dai X, Zuojie L, Zhou A, Meijuan C. Two-year foot care program for minority patients with type 2 diabetes mellitus of Zhuang tribe in Guangxi, China. Can J Diabetes. 2012;36(1):15-8.

39. Li J, Gu L, Guo Y. An educational intervention on foot self-care behaviour among diabetic retinopathy patients with visual disability and their primary caregivers. J Clin Nurs. 2019;28(13-14):2506-16.

40. Lavery LA, LaFontaine J, Higgins KR, Lanctot DR, Constantinides G. Shearreducing insoles to prevent foot ulceration in high-risk diabetic patients. Adv Skin Wound Care. 2012;25(11):519-24.

41. Rönnemaa T, Hämäläinen $H$, Toikka T, Liukkonen I. Evaluation of the impact of podiatrist care in the primary prevention of foot problems in diabetic subjects. Diabetes Care. 1997:20(12):1833-7.

42. Searle A, Spink MJ, Oldmeadow C, Chiu S, Chuter VH. Calf muscle stretching is ineffective in increasing ankle range of motion or reducing plantar pressures in people with diabetes and ankle equinus: a randomised controlled trial. Clin Biomech. 2019;69:52-7.

43. Fan L, Sidani S, Cooper-Brathwaite A, Metcalfe K. Improving foot self-care knowledge, self-efficacy, and behaviors in patients with type 2 diabetes at low risk for foot ulceration: a pilot study. Clin Nurs Res. 2014;23(6):627-43.

44. Health BCMo. Self-managaement support: A health care intervention 2011 [Available from: https://www.selfmanagementbc.ca/uploads/What\%20is\%2 OSelf-Management/PDF/Self-Management\%20Support\%20A\%20health\%2 Ocare\%20intervention\%202011.pdf.

45. Chin YF, Liang J, Wang WS, Hsu BRS, Huang TT. The role of foot self-care behavior on developing foot ulcers in diabetic patients with peripheral neuropathy: a prospective study. Int J Nurs Stud. 2014;51(12):1568-74.

46. Fan L. Examining the feasibility, acceptability and effects of a foot self-care educational intervention in adult patients with diabetes at low risk for foot ulceration: University of Toronto (Canada); 2012.

\section{Publisher's Note}

Springer Nature remains neutral with regard to jurisdictional claims in published maps and institutional affiliations. 